\title{
Hidden charm states with heavy quark spin symmetry in the local hidden gauge approach
}

\author{
Chu-Wen Xiao*市 \\ Departamento de Física Teórica and IFIC, Centro Mixto Universidad de Valencia-CSIC, \\ Institutos de Investigación de Paterna, Apartado 22085, 46071 Valencia, Spain \\ E-mail: Riaochwdific.uv.es
}

\section{Juan Nieves}

IFIC, Centro Mixto Universidad de Valencia-CSIC, Institutos de Investigación de Paterna, Apartado 22085, 46071 Valencia, Spain

E-mail: Gmnievesdific.uv.es

\section{Eulogio Oset}

Departamento de Física Teórica and IFIC, Centro Mixto Universidad de Valencia-CSIC, Institutos de Investigación de Paterna, Apartado 22085, 46071 Valencia, Spain

E-mail: bseteific.uv.es

In this talk, we investigate the meson baryon interaction with hidden charm by exploring a coupled channel unitary approach and using the constraints of heavy quark spin symmetry. By using heavy quark spin symmetry and the dynamics of the local hidden gauge, we obtain four basic states of hidden charm, which are bound by about $50 \mathrm{MeV}$ with respect to the corresponding $\bar{D} \Sigma_{c}, \bar{D} \Sigma_{c}^{*}$, $\bar{D}^{*} \Sigma_{c}$ and $\bar{D}^{*} \Sigma_{c}^{*}$ thresholds respectively and decay mostly into $\eta_{c} N$ and $J / \psi N$. All the states appear in isospin $I=1 / 2$, some of which are degenerate in different angular momentum $J$.

XV International Conference on Hadron Spectroscopy-Hadron 2013

4-8 November 2013

Nara, Japan

\footnotetext{
${ }^{*}$ Speaker.

${ }^{\dagger}$ Thanks for the local support of the organizer.
} 


\section{Introduction}

The topic of baryonic molecules with charm and hidden charm has attracted much attention, and the coming of the FAIR facility is certainly stimulating much work along these lines. In Refs. [U, ㅁ] ], hidden charm baryons were studied, mostly by means of the $\bar{D} \Sigma_{c}, \bar{D} \Lambda_{c}$ and $\bar{D}^{*} \Sigma_{c}, \bar{D}^{*} \Lambda_{c}$ components, and a series of meson-baryon dynamically generated, relatively narrow $N^{*}$ and $\Lambda^{*}$ resonances, were predicted around $4.3 \mathrm{GeV}$. The interaction for the meson-baryon used in Refs. [U, ㅁ] was obtained from an extrapolation to $\mathrm{SU}(4)$, conveniently broken, of the local hidden gauge dynamics used for SU(3) [3], 田, []]. Yet, an element missing in principle in these works is the consideration of heavy quark spin symmetry (HQSS). From the point of view of HQSS, which is a proper QCD spin-flavour symmetry [ $6, \square]$ when the quark masses become much larger than the typical confinement scale, $\Lambda_{\mathrm{QCD}}$, one should consider on the same footing $D$ and $D^{*}$, as well as charmed members of the $20 \mathrm{SU}(4)$ representation of baryons containing the octet of the proton and the 20 representation containing the decuplet of the $\Delta$ when their isospin and strangeness contents

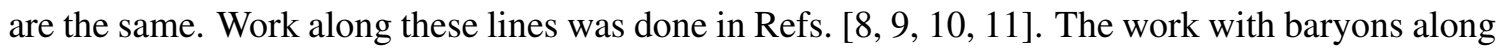

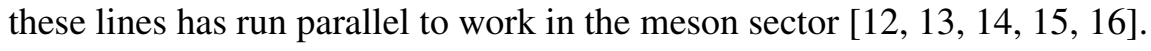

In our work, we use the coupled channel approach to investigate the interaction of mesons and baryons, with the system containing a $c \bar{c}$ component, which can come from pseudoscalar-baryon or vector-baryon interactions. We explore the local hidden gauge approach and introduce some coupled channels, as demanded by HQSS, to study the hidden charm baryons. We obtain several bound states with hidden charm.

\section{Formalism}

We study meson-baryon systems with hidden charm and spin, isospin $I=1 / 2,3 / 2, J=$ $1 / 2,3 / 2,5 / 2$. For the different $I, J$ quantum numbers we have the following space states.

(1) $J=1 / 2, I=1 / 2: \quad \eta_{c} N, J / \psi N, \bar{D} \Lambda_{c}, \bar{D} \Sigma_{c}, \bar{D}^{*} \Lambda_{c}, \bar{D}^{*} \Sigma_{c}, \bar{D}^{*} \Sigma_{c}^{*}$.

(2) $J=1 / 2, I=3 / 2: \quad J / \psi \Delta, \bar{D} \Sigma_{c}, \bar{D}^{*} \Sigma_{c}, \bar{D}^{*} \Sigma_{c}^{*}$.

(3) $J=3 / 2, I=1 / 2: \quad J / \psi N, \bar{D}^{*} \Lambda_{c}, \bar{D}^{*} \Sigma_{c}, \bar{D} \Sigma_{c}^{*}, \bar{D}^{*} \Sigma_{c}^{*}$.

(4) $J=3 / 2, I=3 / 2: \quad \eta_{c} \Delta, J / \psi \Delta, \bar{D}^{*} \Sigma_{c}, \bar{D} \Sigma_{c}^{*}, \bar{D}^{*} \Sigma_{c}^{*}$.

(5) $J=5 / 2, I=1 / 2: \quad \bar{D}^{*} \Sigma_{c}^{*}$.

(6) $J=5 / 2, I=3 / 2: \quad J / \psi \Delta, \bar{D}^{*} \Sigma_{c}^{*}$.

Exploring the HQSS basis we will classify the states in terms of the quantum numbers: $J$, total spin of the meson-baryon system; $\mathscr{L}$, total spin of the light quarks system; $S_{c \bar{c}}$, total spin of the $c \bar{c}$ subsystem; $\ell_{M}$, total spin of the light quarks in the meson; and $\ell_{B}$, total spin of the light quarks in the baryon. The approximate HQSS of QCD leads (neglecting $\mathscr{O}\left(\Lambda_{Q C D} / m_{Q}\right)$ corrections) to important simplifications when the HQSS basis is used (more details seen in Ref. [ㅁ] ]):

$$
\begin{aligned}
& \left(\ell_{M}^{\prime}, \ell_{B}^{\prime}\right) \\
= & \left.\delta_{\alpha \alpha^{\prime}} \delta_{J J^{\prime}}^{\prime} \delta_{S_{c \bar{c}}^{\prime} S_{c \bar{c}}}, \mathscr{L}_{\mathscr{L}}^{\prime} ; \mathscr{L}^{\prime}, \alpha^{\prime}\left|\ell_{M}^{Q C D} \ell_{B}^{\prime} S_{c \bar{c}}, \mathscr{L} ; \alpha \| H^{Q C D}\right| \ell_{M} \ell_{B} \mathscr{L} ; \alpha\right\rangle,
\end{aligned}
$$

where $\alpha$ stands for other quantum numbers (isospin and hypercharge). Note that the reduced matrix elements do not depend on $S_{c \bar{c}}$, because QCD dynamics is invariant under separate spin rotations of the charm quark and antiquark. 


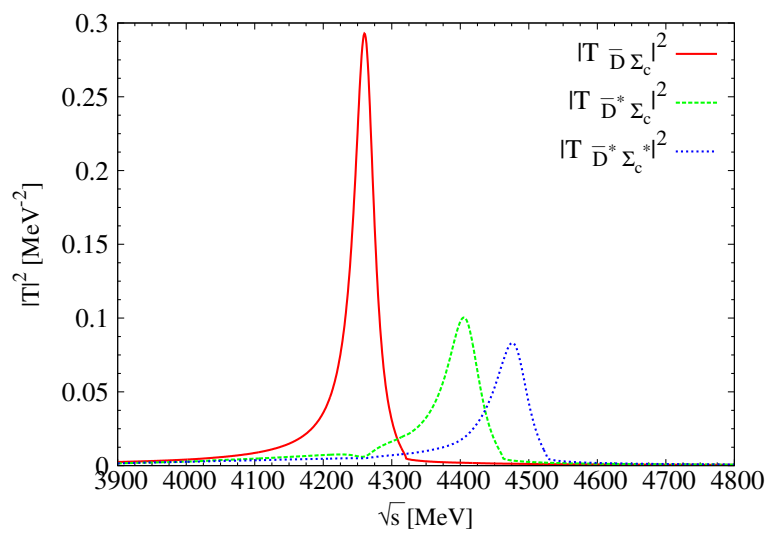

Figure 1: The squared amplitudes of the $J=1 / 2, I=1 / 2$ sector.

\section{Results}

We show the results for the $J=1 / 2, I=1 / 2$ sector in Fig. 四, in terms of the squared amplitudes of $|T|^{2}$. From Fig. 四, we can see three clear peaks with a certain width around the energy range $4200 \sim 4500 \mathrm{MeV}$, which are a little below the thresholds of $\bar{D} \Sigma_{c}, \bar{D}^{*} \Sigma_{c}, \bar{D}^{*} \Sigma_{c}^{*}$ respectively. The poles for these peaks in the second Riemann sheet are found at $(4261.87-$ i17.84) MeV, (4410.13-i29.44) MeV, (4481.35 - i28.91) MeV. The couplings to the various coupled channels for these poles are given in Table $\square$. From Table $\square$ we can conclude that the three

Table 1: The coupling constants of all channels corresponding to certain poles in the $J=1 / 2, I=1 / 2$ sector.

\begin{tabular}{|c|c|c|c|c|c|c|c|}
\hline \multicolumn{8}{|c|}{$4261.87-i 17.84$} \\
\hline & $\eta_{c} N$ & $J / \psi N$ & $\bar{D} \Lambda_{c}$ & $\bar{D} \Sigma_{c}$ & $\bar{D}^{*} \Lambda_{c}$ & $\bar{D}^{*} \Sigma_{c}$ & $\bar{D}^{*} \Sigma_{c}^{*}$ \\
\hline$g_{i}$ & $1.04+i 0.05$ & $0.76-i 0.08$ & $0.02-i 0.02$ & $3.12-i 0.25$ & $0.14-i 0.48$ & $0.33-i 0.68$ & $0.16-i 0.28$ \\
\hline$\left|g_{i}\right|$ & 1.05 & 0.76 & 0.02 & 3.13 & 0.50 & 0.75 & 0.32 \\
\hline \multicolumn{8}{|c|}{$4410.13-i 29.44$} \\
\hline & $\eta_{c} N$ & $J / \psi N$ & $\bar{D} \Lambda_{c}$ & $\bar{D} \Sigma_{c}$ & $\bar{D}^{*} \Lambda_{c}$ & $\bar{D}^{*} \Sigma_{c}$ & $\bar{D}^{*} \Sigma_{c}^{*}$ \\
\hline$g_{i}$ & $0.34+i 0.16$ & $1.43-0.12$ & $0.15-i 0.10$ & $0.20-i 0.05$ & $0.17-i 0.11$ & $3.05-i 0.54$ & $0.07-i 0.51$ \\
\hline$\left|g_{i}\right|$ & 0.38 & 1.44 & 0.18 & 0.20 & 0.20 & 3.10 & 0.51 \\
\hline \multicolumn{8}{|c|}{$4481.35-i 28.91$} \\
\hline & $\eta_{c} N$ & $J / \psi N$ & $\bar{D} \Lambda_{c}$ & $\bar{D} \Sigma_{c}$ & $\bar{D}^{*} \Lambda_{c}$ & $\bar{D}^{*} \Sigma_{c}$ & $\bar{D}^{*} \Sigma_{c}^{*}$ \\
\hline$g_{i}$ & $1.15-i 0.04$ & $0.72+i 0.03$ & $0.18-i 0.08$ & $0.10-i 0.03$ & $0.09-i 0.08$ & $0.09-i 0.06$ & $2.88-i 0.57$ \\
\hline$\left|g_{i}\right|$ & 1.15 & 0.72 & 0.19 & 0.10 & 0.12 & 0.11 & 2.93 \\
\hline
\end{tabular}

poles couple mostly to $\bar{D} \Sigma_{c}, \bar{D}^{*} \Sigma_{c}, \bar{D}^{*} \Sigma_{c}^{*}$ respectively, and decay mostly in $\eta_{c} N$ and $J / \psi N$. The first two states correspond to those reported in Refs. [四, []], and also the first one, $\bar{D} \Sigma_{c}$ state, is consistent with the results using different models [ㅁ, [1]].

For the $J=3 / 2, I=1 / 2$ sector, we show our results in Fig. ‥ From the results of $|T|^{2}$, 


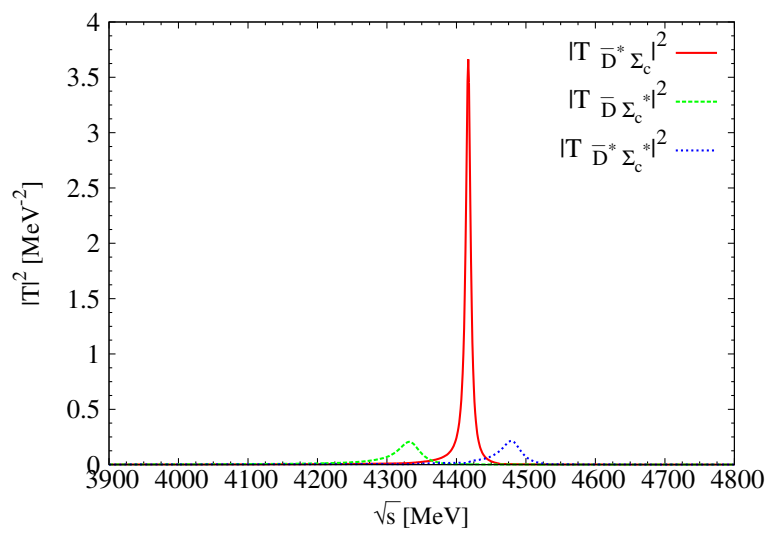

Figure 2: The results of $|T|^{2}$ for the $J=3 / 2, I=1 / 2$ sector. To the right the two small peaks of the left figure magnified.

we can also find three clear peaks around the range $4300 \sim 4500 \mathrm{MeV}$, which are not far below the thresholds of $\bar{D} \Sigma_{c}^{*}, \bar{D}^{*} \Sigma_{c}, \bar{D}^{*} \Sigma_{c}^{*}$ respectively. We look for the poles in the second Riemann sheet, and obtain (4334.45-i19.41) MeV, (4417.04-i4.11) MeV, (4481.04-i17.38) MeV. The couplings to each coupled channel corresponding to these poles are listed in Table \. From Table \,

Table 2: The coupling constants to various channels for certain poles in the $J=3 / 2, I=1 / 2$ sector.

\begin{tabular}{cccccc}
\hline \hline $4334.45-i 19.41$ & $J / \psi N$ & $\bar{D}^{*} \Lambda_{c}$ & $\bar{D}^{*} \Sigma_{c}$ & $\bar{D} \Sigma_{c}^{*}$ & $\bar{D}^{*} \Sigma_{c}^{*}$ \\
\hline$g_{i}$ & $1.31-i 0.18$ & $0.16-i 0.23$ & $0.20-i 0.48$ & $2.97-i 0.36$ & $0.24-i 0.76$ \\
$\left|g_{i}\right|$ & 1.32 & 0.28 & 0.52 & 2.99 & 0.80 \\
\hline $4417.04-i 4.11$ & $J / \psi N$ & $\bar{D}^{*} \Lambda_{c}$ & $\bar{D}^{*} \Sigma_{c}$ & $\bar{D} \Sigma_{c}^{*}$ & $\bar{D}^{*} \Sigma_{c}^{*}$ \\
\hline$g_{i}$ & $0.53-i 0.07$ & $0.08-i 0.07$ & $2.81-i 0.07$ & $0.12-i 0.10$ & $0.11-i 0.51$ \\
$\left|g_{i}\right|$ & 0.53 & 0.11 & 2.81 & 0.16 & 0.52 \\
\hline $4481.04-i 17.38$ & $J / \psi N$ & $\bar{D}^{*} \Lambda_{c}$ & $\bar{D}^{*} \Sigma_{c}$ & $\bar{D} \Sigma_{c}^{*}$ & $\bar{D}^{*} \Sigma_{c}^{*}$ \\
\hline$g_{i}$ & $1.05+i 0.10$ & $0.18-i 0.09$ & $0.12-i 0.10$ & $0.22-i 0.05$ & $2.84-i 0.34$ \\
$\left|g_{i}\right|$ & 1.05 & 0.20 & 0.16 & 0.22 & 2.86 \\
\hline
\end{tabular}

we find that the three poles couple most strongly to the channel $\bar{D} \Sigma_{c}^{*}, \bar{D}^{*} \Sigma_{c}$, and $\bar{D}^{*} \Sigma_{c}^{*}$ respectively. All of them also decay mostly into $J / \psi N$.

Finally, we also obtain a new bound state of $\bar{D}^{*} \Sigma_{c}^{*}$ around $(4487.10-i 0) \mathrm{MeV}$ in the $J=$ $5 / 2, I=1 / 2$ sector, seen in Fig. [3], which we can see that the state has no width, for that there is only a single channel in this sector.

\section{Conclusions}

In present work, we predict seven states with some of them appearing at about the same energy but different $J$. Then, we get states degenerate in $J=1 / 2,3 / 2$ for $\bar{D}^{*} \Sigma_{c}$ and $J=1 / 2,3 / 2,5 / 2$ for $\bar{D}^{*} \Sigma_{c}^{*}$. From this perspective we can report our results that we get four bound states with about 


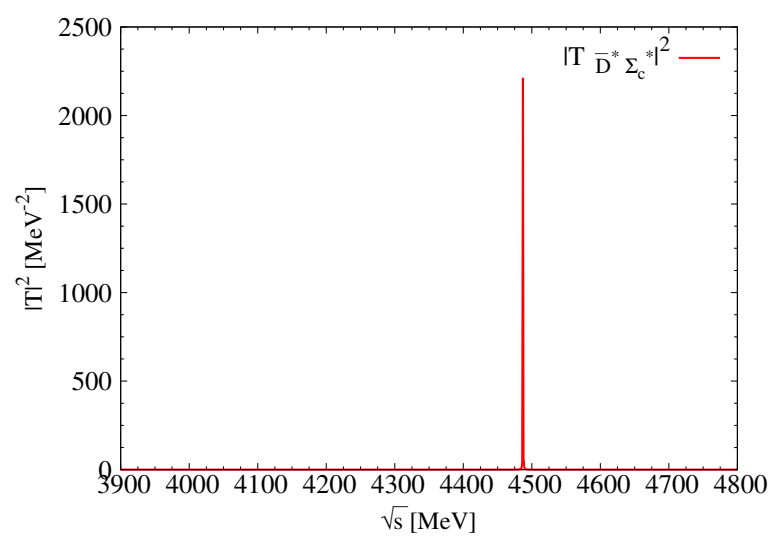

Figure 3: The results of $|T|^{2}$ for the $J=5 / 2, I=1 / 2$ sector.

$40-50 \mathrm{MeV}$ binding, corresponding to $\bar{D} \Sigma_{c}, \bar{D} \Sigma_{c}^{*}$ with $J=1 / 2,3 / 2$ respectively, $\bar{D}^{*} \Sigma_{c}$ degenerate with $J=1 / 2,3 / 2$ and $\bar{D}^{*} \Sigma_{c}^{*}$ degenerate with $J=1 / 2,3 / 2,5 / 2$.

\section{References}

[1] J. -J. Wu, R. Molina, E. Oset and B. S. Zou, Phys. Rev. Lett. 105, 232001 (2010).

[2] J. -J. Wu, R. Molina, E. Oset and B. S. Zou, Phys. Rev. C 84, 015202 (2011).

[3] M. Bando, T. Kugo, S. Uehara, K. Yamawaki and T. Yanagida, Phys. Rev. Lett. 54, 1215 (1985).

[4] M. Bando, T. Kugo and K. Yamawaki, Phys. Rept. 164, 217 (1988).

[5] U. G. Meissner, Phys. Rept. 161, 213 (1988).

[6] N. Isgur and M. B. Wise, Phys. Lett. B 232, 113 (1989).

[7] M. Neubert, Phys. Rept. 245, 259 (1994).

[8] C. Garcia-Recio, V. K. Magas, T. Mizutani, J. Nieves, A. Ramos, L. L. Salcedo and L. Tolos, Phys. Rev. D 79, 054004 (2009).

[9] D. Gamermann, C. Garcia-Recio, J. Nieves, L. L. Salcedo and L. Tolos, Phys. Rev. D 81, 094016 (2010).

[10] O. Romanets, L. Tolos, C. Garcia-Recio, J. Nieves, L. L. Salcedo and R. G. E. Timmermans, Phys. Rev. D 85, 114032 (2012).

[11] C. Garcia-Recio, J. Nieves, O. Romanets, L. L. Salcedo and L. Tolos, Phys. Rev. D 87, 074034 (2013).

[12] J. Nieves and M. P. Valderrama, Phys. Rev. D 86, 056004 (2012).

[13] C. Hidalgo-Duque, J. Nieves and M. P. Valderrama, Phys. Rev. D 87, 076006 (2013).

[14] C. Hidalgo-Duque, J. Nieves and M. PavÃşn Valderrama, Nucl. Phys. A 914, 482 (2013).

[15] F. -K. Guo, C. Hanhart and U. -G. Meissner, Phys. Rev. Lett. 102, 242004 (2009).

[16] F. -K. Guo, C. Hidalgo-Duque, J. Nieves and M. P. Valderrama, Phys. Rev. D 88, 054007 (2013).

[17] C. W. Xiao, J. Nieves and E. Oset, Phys. Rev. D 88, 056012 (2013).

[18] J. -J. Wu, T. -S. H. Lee and B. S. Zou, Phys. Rev. C 85, 044002 (2012).

[19] W. L. Wang, F. Huang, Z. Y. Zhang and B. S. Zou, Phys. Rev. C 84, 015203 (2011). 